\title{
Extracorporeal life support to ventricular assist device: potential benefits of sternal-sparing approach
}

\author{
Fabio M. Sagebin ${ }^{1 \#}$, Brian C. Ayers ${ }^{1 \#}$, Katherine Wood ${ }^{1}$, Bryan Barrus ${ }^{1}$, Leway Chen ${ }^{2}$, Jeffrey Alexis ${ }^{2}$, \\ Himabindu Vidula ${ }^{2}$, Sabu Thomas ${ }^{2}$, Eugene Storozynsky ${ }^{2}$, Sunil Prasad ${ }^{1}$, Igor Gosev ${ }^{1}$ \\ ${ }^{1}$ Division of Cardiac Surgery, ${ }^{2}$ Division of Cardiology, University of Rochester Medical Center, Rochester, NY, USA \\ Contributions: (I) Conception and design: I Gosev, F Sagebin, K Wood, B Ayers; (II) Administrative support: S Prasad; (III) Provision of study \\ materials or patients: I Gosev, B Barrus, S Prasad; (IV) Collection and assembly of data: B Ayers, F Sagebin; (V) Data analysis and interpretation: B \\ Ayers, F Sagebin, K Wood, I Gosev; (VI) Manuscript writing: All authors; (VII) Final approval of manuscript: All authors. \\ \#These authors contributed equally to this work. \\ Correspondence to: Igor Gosev, MD. Division of Cardiac Surgery, University of Rochester Medical Center, 601 Elmwood Avenue, Rochester, NY \\ 14642, USA. Email: Igor_gosev@urmc.rochester.edu.
}

Background: Advancements in left ventricular assist device (LVAD) technology have improved long-term survival for properly selected patients with advanced heart failure. However, the subset of patients in critical cardiogenic shock remain difficult to treat with persistently high patient morbidity and mortality. The goal of this study is to describe our institutional experience utilizing extracorporeal membrane oxygenation (ECMO) as a bridge to LVAD for patients in cardiogenic shock comparing the less invasive complete sternal-sparing (CSS) surgical technique to median sternotomy.

Methods: Data was collected as a single center retrospective review of patients implanted with a continuous-flow LVAD directly off ECMO from 2012 to 2018. Patients were stratified by LVAD surgical technique. The primary outcome was survival to discharge. Secondary outcomes included postoperative complications, resource utilization and survival at 6-months.

Results: Of the 37 patients implanted directly off ECMO, 26 (70\%) patients were implanted via median sternotomy and 11 (30\%) patients by the CSS approach. Median time on ECMO support was 8 days (range, 2-29 days). Preoperative characteristics were similar between groups. Survival to discharge was 78\% overall (73\% vs. 91\% CSS, $\mathrm{P}=0.391$ ). The CSS cohort had fewer postoperative complications, including fewer transfusions $(\mathrm{P}=0.044)$ and trend towards less right ventricular (RV) failure (62\% vs. 27\% CSS, $\mathrm{P}=0.079)$. Both cohorts required similar median length of stay (LOS) in the intensive care unit (ICU) (11 vs. 12 days, $\mathrm{P}=0.695$ ) and similar overall hospital LOS (34 vs. 22 days, $\mathrm{P}=0.242$ ). Overall survival was $74 \%$ at six months (68\% vs. 89\% CSS, $\mathrm{P}=0.386$ ).

Conclusions: VA ECMO can be used effectively as a bridge-to-LVAD for patients in cardiogenic shock. The less invasive CSS approach demonstrates potential advantages to median sternotomy. Further study is needed to better understand the benefits of less invasive surgical techniques.

Keywords: Ventricular assist devices; extracorporeal life support; minimally invasive surgery; cardiogenic shock; operative surgical procedure

Submitted Apr 18, 2019. Accepted for publication Sep 27, 2019.

doi: $10.21037 /$ jtd.2019.10.21

View this article at: http://dx.doi.org/10.21037/jtd.2019.10.21

\section{Introduction}

Improvements in left ventricular assist device (LVAD) technology have produced increasingly positive long-term outcomes for patients with heart failure (1-3). However, patients in critical cardiogenic shock remain difficult to treat and have persistently high patient morbidity and mortality (4). There is significant debate over the 
optimal use of preoperative support and timing of LVAD implantation for this critically ill patient population. Management strategies and outcomes vary significantly between institutions (5-8).

In the modern LVAD era (2012-2017), nearly 30\% of patients are supported with temporary MCS devices preoperatively, however only $2.5 \%$ of patients are supported by extracorporeal membrane oxygenation (ECMO) (9). VA ECMO can provide life sustaining end-organ perfusion, but it often does not address the underlying problem and is associated with a high incidence of complications (10). As such, the use of VA ECMO as a bridge-to-LVAD strategy is highly debated with a wide range of reported outcomes (11-17).

An additional consideration is in the optimal surgical technique to employ in this patient population. Advances in LVAD technology have allowed for implantation via less invasive techniques. Early studies have demonstrated a number of potential advantages of these approaches, including less postoperative bleeding and a lower incidence of severe right ventricular (RV) failure $(18,19)$. INTERMACS-1 patients and those supported by VA ECMO have been shown to be at increased risk for both these complications (20). Therefore, we hypothesized that there would be a substantial benefit in performing less invasive LVAD implantation compared to sternotomy for patients supported by VA ECMO.

The goal of this study is to describe our institutional experience utilizing VA ECMO as bridge-to-LVAD therapy, including a sub-analysis of early outcomes for the less invasive complete sternal-sparing (CSS) surgical technique compared to traditional full sternotomy.

\section{Methods}

\section{Patient selection}

We retrospectively reviewed a prospectively maintained database of patients supported by ECMO at our institution from October 2012 through October 2018. Adult patients that were bridged directly from VA ECMO to a continuous-flow LVAD were included in the study. For the sub-analysis, patients were dichotomized based on surgical approach: traditional median sternotomy (sternotomy cohort) or the less invasive complete-sternal sparing approach (CSS cohort). The study was approved by the institutional ethics board of University of Rochester (No. 00071273).

\section{Study variables}

Patient demographics, comorbidities, and preoperative interventions before LVAD implantation were collected for all patients. Intraoperative data were reviewed including cardiopulmonary bypass $(\mathrm{CPB})$ time, total procedural time, and use of intraoperative blood products. Outcome measures were collected, including intensive care unit (ICU) length of stay (LOS), total hospital LOS, time on mechanical ventilation, blood product utilization, survival to discharge and incidence of severe RV or end organ failure. All postoperative complications were assessed according to INTERMACS definitions, including severe RV failure as defined by the use of right ventricle assist device (RVAD), severe RV dysfunction on echocardiography, or inotropic therapy for longer than 14 days postoperatively.

\section{ECMO initiation and patient selection}

Patients were initiated on VA ECMO either at our institution or transferred from another institution while on VA ECMO. The need for left ventricle (LV) decompression was assessed using transthoracic echocardiography. Patients that exhibited $\mathrm{LV}$ distention were treated with optimizing hemodynamics, transapical cannulation or a percutaneous LVAD (Impella, Abiomed, Danvers, USA). An assessment to determine LVAD eligibility was initiated for all patients stabilized on VA ECMO who had a high probability for meaningful recovery. Specifically, we assessed for signs of irreversible brain damage and monitored laboratory indicators of end organ function. Patients who showed improvement in laboratory values with no signs of irreversible end organ damage and had no other contraindications to LVAD therapy were deemed eligible. There was no predefined cutoff for age (21). This was determined by a multidisciplinary team consisting of cardiac surgeons, intensive care specialists, heart failure cardiologists, and VAD coordinators.

\section{LVAD surgical technique}

Patients underwent LVAD implantation via either traditional median sternotomy or the previously described less invasive CSS approach (19). The CSS approach for LVAD implantation became our standard surgical technique as an institution starting in February 2018. Since that time, every patient has undergone a non-contrast computed tomography (CT) of the chest preoperatively to assess their aortic position. Patients with an aorta positioned to the left of the sternum pose a more difficult aortic exposure via the 
right thoracotomy. For these patients a sternotomy was considered at the discretion of the surgeon. Intraoperatively, both cohorts of patients were transitioned from VA ECMO to $\mathrm{CPB}$ using the VA ECMO cannulas. Both cohorts received the same intraoperative and post-operative goaldirected therapy from the same multidisciplinary team. Patients requiring RVAD were supported with either the CentriMag (Thoratec, Pleasanton, USA) or Protek Duo (Tandem Life, Pittsburgh, USA) (22). Delayed surgical closure was employed at the discretion of the surgeon based

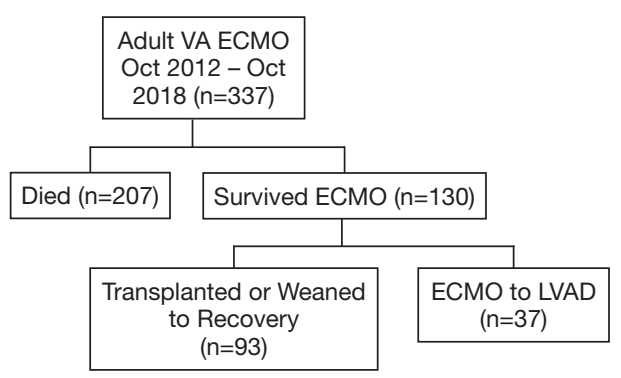

Figure 1 Outcomes of patients supported by VA ECMO. ECMO, extracorporeal membrane oxygenation; LVAD, left ventricular assist device. on either hemodynamic instability when closing the chest, persistent coagulopathic bleeding, or the use of a centrally cannulated RVAD.

\section{Statistical analysis}

Categorical variables are presented as count (percentage) and were compared with the Fisher Exact test. Continuous data are presented as mean $\pm \mathrm{SD}$ or median (IQR). Student's $t$-test and Wilcoxon rank sum test were used to compare normal and non-normally distributed continuous data, respectively. Wilcoxon signed rank test were used to investigate changes in laboratory values, comparing peak values while on ECMO to the immediate preoperative value for each lab. For all analyses, a p-value less than 0.05 was considered significant. Statistical analysis was performed using Stata software (StataCorp. 2015. Stata Statistical Software: Release 14. College Station, USA).

\section{Results}

\section{VA ECMO support}

A total of 337 adult patients were supported by VA ECMO at our institution from October 2012 through October 2018. Of

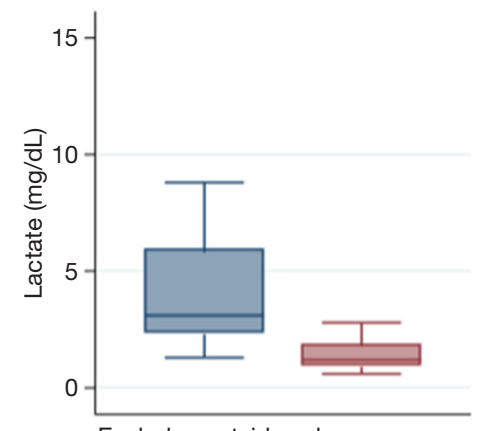

Excludes outside values

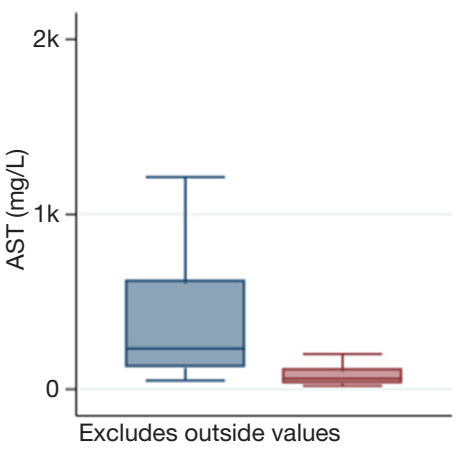

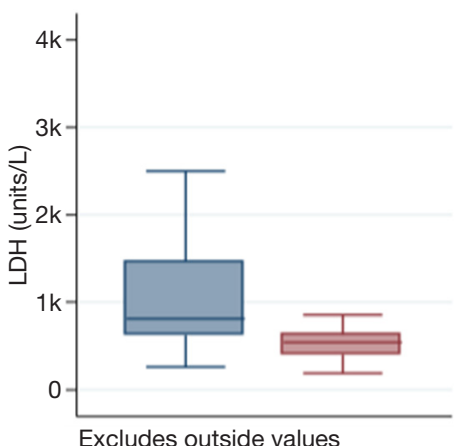

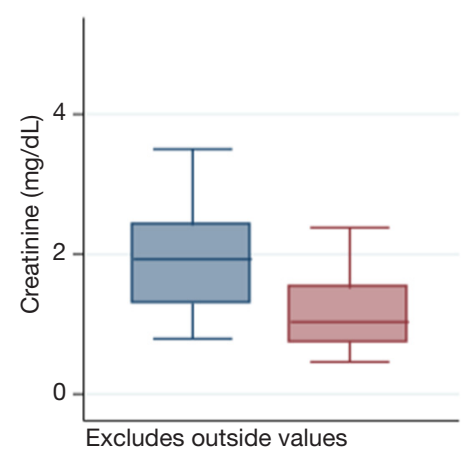

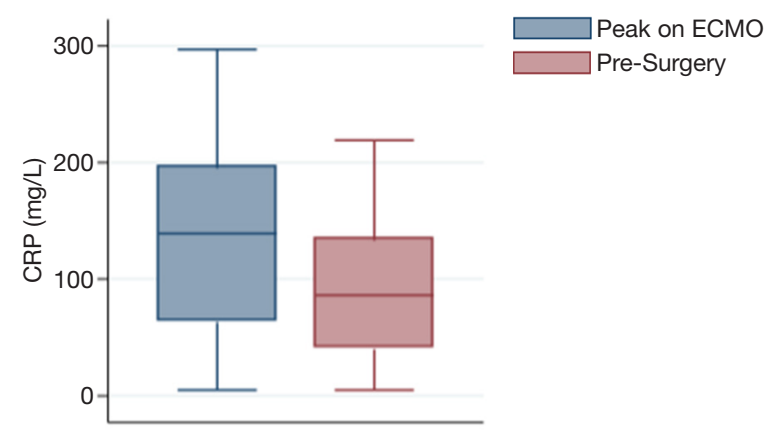

Excludes outside values

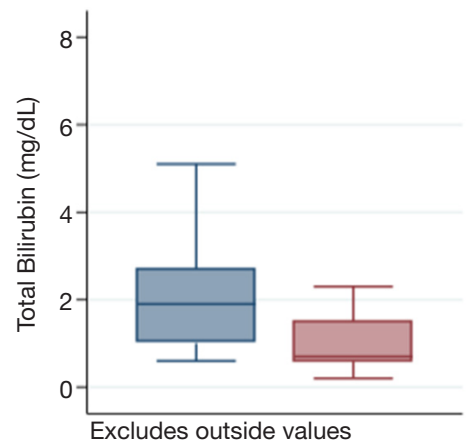

Figure 2 Improvements in laboratory values from VA ECMO support prior to LVAD implantation (signed rank test $\mathrm{P}<0.001$ for all). ECMO, extracorporeal membrane oxygenation; LVAD, left ventricular assist device. 
Table 1 Patient characteristics

\begin{tabular}{|c|c|}
\hline Variable* & ECMO-to-VAD $(n=37)$ \\
\hline \multicolumn{2}{|l|}{ Patient characteristics } \\
\hline Age (years) & 56 [51-62] \\
\hline Male (\%) & 29 [78] \\
\hline White (\%) & 35 [95] \\
\hline $\mathrm{BMI}\left(\mathrm{kg} / \mathrm{m}^{2}\right)$ & $29.35[25.35-34]$ \\
\hline Pre-ECMO cardiac arrest (\%) & $14[38]$ \\
\hline \multicolumn{2}{|l|}{ Past medical history (\%) } \\
\hline Past cardiac procedure & $3[8]$ \\
\hline Ischemic cardiomyopathy & 23 [62] \\
\hline CKD not requiring dialysis & $6[16]$ \\
\hline Diabetes & $10[27]$ \\
\hline COPD & $3[8]$ \\
\hline Arrhythmia & $7[19]$ \\
\hline \multicolumn{2}{|l|}{ Preoperative support (\%) } \\
\hline Inotropes & 27 [73] \\
\hline Impella or IABP & 18 [49] \\
\hline ECMO & $37[100]$ \\
\hline Ventilator & $22[59]$ \\
\hline \multicolumn{2}{|l|}{ Preoperative hemodynamics } \\
\hline LVEF (\%) & $20.1 \pm 10.4$ \\
\hline PCWP (mmHg) & $22.6 \pm 11.0$ \\
\hline Cardiac index $\left(\mathrm{L} / \mathrm{min} / \mathrm{m}^{2}\right)$ & $1.8 \pm 0.5$ \\
\hline Mean PA pressure (mmHg) & $32.6 \pm 7.0$ \\
\hline PVR (wood units) & $3.8 \pm 2.5$ \\
\hline RA pressure (mmHg) & $13.8 \pm 8.8$ \\
\hline $\operatorname{RVSWI}\left(\mathrm{mmHg} / \mathrm{mL} / \mathrm{m}^{2}\right)$ & $355.0 \pm 172.8$ \\
\hline RVSWI <400 (\%) & $6[16]$ \\
\hline \multicolumn{2}{|l|}{ Intended goal (\%) } \\
\hline Bridge to transplant & $26[70]$ \\
\hline Destination therapy & 9 [24] \\
\hline Bridge to recovery & $2[5]$ \\
\hline \multicolumn{2}{|l|}{ Device brand (\%) } \\
\hline HeartMate II & $15[41]$ \\
\hline HeartMate 3 & 18 [49] \\
\hline HeartWare HVAD & $4[11]$ \\
\hline
\end{tabular}

${ }^{*}$, values presented as No. [\%], mean $\pm \mathrm{SD}$, or median [IQR]. ECMO, extracorporeal membrane oxygenation; VAD, ventricular assist device; BMI, body mass index; CKD, chronic kidney disease; COPD, chronic obstructive pulmonary disease; IABP, intra-aortic balloon pump; LVEF, left ventricular ejection fraction; PCWP, pulmonary-capillary wedge pressure; PA, pulmonary artery; PVR, pulmonary vascular resistance; RA, right atrium; PAPI, pulmonary artery pulsatility index.
Table 2 Procedural data

\begin{tabular}{|c|c|}
\hline Variable* $^{\star}$ & ECMO-to-VAD (n=37) \\
\hline \multicolumn{2}{|l|}{ Procedural data } \\
\hline CPB time [min] & $110[83-164]$ \\
\hline Surgery time [min] & $330[240-407]$ \\
\hline Concomitant procedure [\%] & $4[11]$ \\
\hline \multicolumn{2}{|c|}{ Intraoperative blood products (units) } \\
\hline PRBC & $3[1-6]$ \\
\hline FFP & $2[1.5-4]$ \\
\hline Platelets & $1[1-2]$ \\
\hline Cryoprecipitate & $0[0-1]$ \\
\hline Delayed closure [\%] & 24 [65] \\
\hline
\end{tabular}

the 130 (39\%) patients that survived ECMO, 37 (28\%) patients underwent LVAD implantation directly off ECMO (Figure 1). Median time on ECMO support before LVAD implantation was 8 days (range, 2-29 days). The stabilizing effect of ECMO can be seen in the improvement in laboratory values (Figure 2). Laboratory markers of inflammation and end-organ functionincluding lactate, c-reactive protein, liver function tests, and creatinine-demonstrated marked improvement when comparing peak values while on ECMO to the values immediately before LVAD implantation.

\section{Patient characteristics}

Median patient age at time of LVAD implantation was 56 years (IQR, 51-62 years) (Table 1 ). The majority of patients were white (95\%) and male (78\%). A large number of patients experienced cardiac arrest (38\%) before initiation of ECMO. In addition to VA ECMO, the majority of patients required inotropic support (73\%). Many needed mechanical ventilation (59\%) and nearly half (49\%) required Impella or intra-aortic balloon pump (IABP) support during escalation of care before initiation of VA ECMO.

\section{LVAD implantation, complications and early outcomes}

Procedural data are shown in Table 2. The majority of patients $(65 \%)$ required delayed closure due to bleeding 
Table 3 Postoperative complications and early outcomes for LVAD implantation directly off ECMO via CSS compared to median sternotomy approach

\begin{tabular}{|c|c|c|c|c|}
\hline Variable* $^{\star}$ & Total $(\mathrm{n}=37)$ & $\operatorname{CSS}(n=11)$ & Sternotomy $(n=26)$ & $P$ value \\
\hline Discharged home (\%) & 8 [22] & $3[27]$ & $5[19]$ & 0.672 \\
\hline Discharged rehab (\%) & $21[57]$ & $7[64]$ & $14[54]$ & 0.723 \\
\hline Overall complications & $24[65]$ & $5[45]$ & 19 [73] & 0.143 \\
\hline Use of RVAD (\%) & $10[27]$ & $2[18]$ & $8[31]$ & 0.688 \\
\hline Unplanned RTOR (\%) & $6[16]$ & $0[0]$ & $6[23]$ & 0.148 \\
\hline RTOR for bleeding (\%) & $2[5]$ & $0[0]$ & $2[8]$ & 1.000 \\
\hline Respiratory failure (\%) & 23 [62] & $5[45]$ & 18 [69] & 0.268 \\
\hline Pneumonia (\%) & $7[19]$ & $1[9]$ & 6 [23] & 0.649 \\
\hline Surgical site infection (\%) & $3[8]$ & $2[18]$ & $1[4]$ & 1.000 \\
\hline Renal failure requiring dialysis (\%) & 9 [24] & $2[18]$ & $7[27]$ & 0.695 \\
\hline Hepatic failure (\%) & $6[16]$ & $2[18]$ & $4[15]$ & 1.000 \\
\hline \multicolumn{5}{|l|}{ Transfusions within 24 h postop (units) } \\
\hline PRBC & $3[1-7]$ & $1[1-3]$ & $4[2-7]$ & 0.044 \\
\hline FFP & $4[2-7]$ & $2.5[2-4]$ & $4[3-10]$ & 0.045 \\
\hline Platelets & $2[1-3]$ & $2[1-2]$ & $2[1-3]$ & 0.543 \\
\hline Required readmission & $1[3]$ & $0[0]$ & $1[4]$ & 1.000 \\
\hline Six-month survival & $25 / 34[74]$ & $8 / 9$ [89] & $17 / 25[68]$ & 0.386 \\
\hline
\end{tabular}

*, values presented as No. [\%], mean $\pm \mathrm{SD}$, or median [IQR]. LVAD, left ventricular assist device; ECMO, extracorporeal membrane oxygenation; CSS, complete sternal-sparing; RV, right ventricle; RVAD, right ventricular assist device; RTOR, return to operating room; PRBC, packed red blood cells; FFP, fresh frozen plasma; ICU, intensive care unit; LOS, length of stay.

concerns at the conclusion of the operation. Postoperative complications and outcomes are summarized in Table 3. Survival to hospital discharge was $78 \%$ overall. Few patients required an unplanned return to operating room (RTOR) due to complications (16\%), however there was a high incidence of RV failure (51\%) and postoperative respiratory failure (62\%). Median overall hospital LOS was 31 days (IQR,
20-44 days) including an ICU median stay of 11 days (IQR, 5-23 days). Only one (3\%) patient required readmission within 30 days. Overall survival was $74 \%$ at six months.

\section{Surgical approach analysis}

Dichotomizing by surgical approach, 11 (30\%) patients 
were implanted via the CSS approach and 26 (70\%) patients via median sternotomy. The CSS approach for LVAD implantation became our standard surgical technique as an institution starting in February 2018. Since that time all but two of the patients underwent CSS: one was enrolled in a national trial requiring sternotomy and the other had a prior sternotomy during index hospitalization. There were no significant differences in patient preoperative characteristics between the cohorts including age, body mass index (BMI), preoperative hemodynamics, INTERMACS profile, time on ECMO support or intended goal of LVAD therapy (Table S1). Due to our institutional adoption of the less invasive approach in February 2018, the CSS cohort was more commonly implanted with the Heartmate 3 (HM3) (91\% vs. 31\%, $\mathrm{P}=0.001$ ) while the sternotomy cohort more frequently received the Heartmate II (HMII) device (58\% vs. $0 \%, \mathrm{P}=0.001$ ). Early outcomes demonstrate $91 \%$ survival to discharge for the CSS patients compared to $73 \%$ for the sternotomy group ( $\mathrm{P}=0.391$, Table 3$)$. The CSS cohort demonstrated a trend towards fewer cases of severe RV failure $(27 \%$ vs. $62 \%, \mathrm{P}=0.079)$. CSS patients also required less time on mechanical ventilation postoperatively $(\mathrm{P}=0.012)$ and significantly fewer postoperative blood transfusions $(\mathrm{P}=0.044)$. There was no difference between the groups in ICU or hospital LOS.

\section{Discussion}

In this study we aim to describe our experience using VA ECMO to bridge cardiogenic shock patients to continuousflow LVAD, including early outcomes for patients implanted via the less invasive sternal-sparing approach. Our study cohort consisted of critically ill patients with $38 \%$ experiencing cardiac arrest and half requiring Impella or IABP support concurrently with VA ECMO. Patients were supported by ECMO for a median 8 days (range, 2-29 days) preoperatively with marked improvement in their laboratory markers of end organ function. Despite their unfavorable initial risk profile, this strategy resulted in $78 \%$ overall survival to discharge, including $91 \%$ survival for patients implanted via the CSS approach.

The optimal management for critically ill patients in cardiogenic remains controversial with a wide range of strategies and reported outcomes (11-17). Our results support previous studies that have demonstrated high survival rates when utilizing VA ECMO as a bridge-toLVAD (11-14). Our goal directed therapy focuses on proper patient selection by ensuring evidence of end-organ recovery during ECMO support, adequate decompression of the heart with liberal congruent use of an IABP or Impella when necessary, and implanting appropriate LVAD candidates as soon as possible to minimize the amount of time on ECMO support preoperatively $(13,16)$.

In regard to less invasive LVAD surgical techniques, the CSS cohort demonstrated evidence of fewer postoperative complications; including fewer postoperative blood transfusions and a trend towards lower incidence of RV failure compared to median sternotomy. This finding supports previous work that showed a similar reduction in $\mathrm{RV}$ failure and fewer reoperations for bleeding when using a hemisternotomy compared to full sternotomy in cardiogenic shock patients (18). Minimizing the incidence of severe RV failure is crucial for INTERMACS-1 patients who have been shown to have a greater than 2 -fold increased risk for postoperative RV failure compared to all other profiles (20). A potential mechanism for this observed decrease in RV failure includes preservation of the pericardium over the right ventricle and avoidance of right coronary kinking by maintaining the anatomical position of the heart throughout the procedure when using the less invasive approach. Previous studies have shown the pericardium is essential for maintaining RV function during times of acutely increased RV diastolic pressure (23), a common hemodynamic state after LVAD implantation. When using the CSS approach, the pericardium is opened only over the apex and ascending aorta, preserving the pericardium's physiologic constraint over the entirety of the RV. A recent prospective, multicenter study demonstrated improved outcomes for coronary artery bypass graft (CABG) patients that underwent pericardial closure using a decellularized extracellular matrix graft, including fewer pericardial effusions, fewer postoperative arrhythmias, and fewer 30-day readmissions compared to patients with an unrepaired pericardiotomy (24). More research is needed into the role of the pericardium in LVAD recipients.

Previous studies have described favorable survival using emergent LVAD implantation instead of ECMO as the primary therapy for cardiogenic shock (25). However, this strategy does not allow time for a potential full cardiac recovery without needing to undergo durable mechanical circulatory support (MCS) implantation. Moreover, it requires rapid screening for LVAD eligibility, which can result in LVAD implantation in patients who are subsequently found to be inappropriate candidates. VA ECMO as a bridge allows time for end-organ recovery and for a thorough evaluation of each patient. In the present 
study, only $28 \%$ of VA ECMO survivors required durable LVAD support, with the rest experiencing full recovery or proceeding directly to transplantation. Moreover, many of our patients on VA ECMO are stabilized enough that sedation can be weaned, and the patient extubated to allow for a goals of care discussion directly with the patient prior to proceeding with the life altering decision to undergo LVAD implantation.

There are limitations to this study that must be addressed. Given the retrospective nature of the study there is an inherent risk for patient selection bias. Patients were not randomized to either pump model nor surgical technique which could have been impacted by surgeon bias. While all implants were done at a single institution, changes to personnel and standards of care over the study period may also be confounding factors. Furthermore, this is a single center experience that may not generalize to other institutions.

\section{Conclusions}

In conclusion, our results support the utilization of VA ECMO as a bridge-to-LVAD in patients with critical cardiogenic shock. Early data utilizing the less invasive CSS approach demonstrates a $91 \%$ survival to discharge and potential advantages compared to traditional sternotomy, including fewer transfusions. Further study is needed to better understand the potential benefits of less invasive surgical techniques for patients in cardiogenic shock.

\section{Acknowledgments}

We would like to acknowledge our ECMO Specialists and VAD team for their support and contribution to this project.

\section{Footnote}

Conflicts of Interest: Igor Gosev is a consultant for Abbott. Sunil Prasad is a member of the scientific advisory board for Abbott. The other authors have no conflicts of interest to declare.

Ethical Statement: The authors are accountable for all aspects of the work in ensuring that questions related to the accuracy or integrity of any part of the work are appropriately investigated and resolved. The study was approved by the institutional ethics board of University of
Rochester (No. 00071273).

\section{References}

1. Slaughter MS, Rogers JG, Milano CA, et al. Advanced heart failure treated with continuous-flow left ventricular assist device. N Engl J Med 2009;361:2241-51.

2. Mehra MR, Naka Y, Uriel N, et al. A Fully Magnetically Levitated Circulatory Pump for Advanced Heart Failure. N Engl J Med 2017;376:440-50.

3. Mehra MR, Goldstein DJ, Uriel N, et al. TwoYear Outcomes with a Magnetically Levitated Cardiac Pump in Heart Failure. N Engl J Med 2018;378:1386-95.

4. Harjola VP, Lassus J, Sionis A, et al. Clinical picture and risk prediction of short-term mortality in cardiogenic shock. Eur J Heart Fail 2015;17:501-9.

5. Csepe TA, Kilic A. Advancements in mechanical circulatory support for patients in acute and chronic heart failure. J Thorac Dis 2017;9:4070-83.

6. Thiele H, Zeymer U, Neumann FJ, et al. Intra-aortic balloon counterpulsation in acute myocardial infarction complicated by cardiogenic shock (IABP-SHOCK II): final 12 month results of a randomised, open-label trial. Lancet 2013;382:1638-45.

7. den Uil CA, Akin S, Jewbali LS, et al. Short-term mechanical circulatory support as a bridge to durable left ventricular assist device implantation in refractory cardiogenic shock: a systematic review and meta-analysis. Eur J Cardiothorac Surg 2017;52:14-25.

8. Morici N, Oliva F, Ajello S, et al. Management of cardiogenic shock in acute decompensated chronic heart failure: The ALTSHOCK phase II clinical trial. Am Heart J 2018;204:196-201.

9. Kormos RL, Cowger J, Pagani FD, et al. The Society of Thoracic Surgeons Intermacs Database Annual Report: Evolving Indications, Outcomes, and Scientific Partnerships. Ann Thorac Surg 2019;107:341-53.

10. Baran DA. Extracorporeal Membrane Oxygenation (ECMO) and the Critical Cardiac Patient. Curr Transplant Rep 2017;4:218-25.

11. Han JJ, Chung J, Chen CW, et al. Different Clinical Course and Complications in Interagency Registry for Mechanically Assisted Circulatory Support 1 (INTERMACS) Patients Managed With or Without Extracorporeal Membrane Oxygenation. ASAIO J 2018;64:318-22.

12. Riebandt J, Haberl T, Mahr S, et al. Preoperative patient 
optimization using extracorporeal life support improves outcomes of INTERMACS Level I patients receiving a permanent ventricular assist device. Eur J Cardiothorac Surg 2014;46:486-92; discussion 492.

13. Durinka JB, Bogar LJ, Hirose H, et al. End-organ recovery is key to success for extracorporeal membrane oxygenation as a bridge to implantable left ventricular assist device. ASAIO J 2014;60:189-92.

14. Schibilsky D, Haller C, Lange B, et al. Extracorporeal life support prior to left ventricular assist device implantation leads to improvement of the patients INTERMACS levels and outcome. PLoS One 2017;12:e0174262.

15. Shah P, Smith S, Haft JW, et al. Clinical Outcomes of Advanced Heart Failure Patients with Cardiogenic Shock Treated with Temporary Circulatory Support Before Durable LVAD Implant. ASAIO J 2016;62:20-7.

16. Tsyganenko D, Gromann TW, Schoenrath F, et al. Predictors of mid-term outcomes in patients undergoing implantation of a ventricular assist device directly after extracorporeal life support. Eur J Cardiothorac Surg 2019;55:773-9.

17. Kurihara C, Kawabori M, Sugiura T, et al. Bridging to a Long-Term Ventricular Assist Device With ShortTerm Mechanical Circulatory Support. Artif Organs 2018;42:589-96.

18. Wert L, Chatterjee A, Dogan G, et al. Minimally invasive surgery improves outcome of left ventricular assist device surgery in cardiogenic shock. J Thorac Dis
2018;10:S1696-702.

19. Wood KL, Ayers BC, Sagebin F, et al. Complete SternalSparing HeartMate 3 Implantation: A Case Series of 10 Consecutive Patients. Ann Thorac Surg 2019;107:1160-5.

20. Kirklin JK, Pagani FD, Kormos RL, et al. Eighth annual INTERMACS report: Special focus on framing the impact of adverse events. J Heart Lung Transplant 2017;36:1080-6.

21. Kilic A, Sultan I, Yuh DD, et al. Ventricular assist device implantation in the elderly: nationwide outcomes in the United States. J Card Surg 2013;28:183-9.

22. Sultan I, Kilic A, Kilic A. Short-Term Circulatory and Right Ventricle Support in Cardiogenic Shock: Extracorporeal Membrane Oxygenation, Tandem Heart, CentriMag, and Impella. Heart Fail Clin 2018;14:579-83.

23. Burger W, Straube M, Behne M, et al. Role of pericardial constraint for right ventricular function in humans. Chest 1995;107:46-9.

24. Rego A, Cheung PC, Harris WJ, et al. Pericardial closure with extracellular matrix scaffold following cardiac surgery associated with a reduction of postoperative complications and 30-day hospital readmissions. J Cardiothorac Surg 2019;14:61.

25. Pawale A, Schwartz Y, Itagaki S, et al. Selective implantation of durable left ventricular assist devices as primary therapy for refractory cardiogenic shock. J Thorac Cardiovasc Surg 2018;155:1059-68.
Cite this article as: Sagebin FM, Ayers BC, Wood K, Barrus B, Chen L, Alexis J, Vidula H, Thomas S, Storozynsky E, Prasad S, Gosev I. Extracorporeal life support to ventricular assist device: potential benefits of sternal-sparing approach. J Thorac Dis 2019;11(11):4790-4797. doi: 10.21037/jtd.2019.10.21 
Table S1 Patient characteristics for patients implanted with LVAD directly off ECMO via the CSS approach compared to median sternotomy

\begin{tabular}{|c|c|c|c|c|}
\hline Variable* & Total $(n=37)$ & $\operatorname{CSS}(n=11)$ & Sternotomy $(n=26)$ & $P$ value \\
\hline \multicolumn{5}{|l|}{ VA ECMO support } \\
\hline Time on ECMO [days] & 8 [4-13] & $7[3-13]$ & $8[4-15]$ & 0.661 \\
\hline Pre-ECMO cardiac arrest [\%] & $14[38]$ & $3[27]$ & $11[42]$ & 0.477 \\
\hline \multicolumn{5}{|l|}{ Patient characteristics } \\
\hline Age [years] & 56 [51-62] & 55 [52-62] & 56 [51-62] & 0.770 \\
\hline Male [\%] & 29 [78] & 8 [73] & $21[81]$ & 0.672 \\
\hline White [\%] & 35 [95] & $11[100]$ & 24 [92] & 1.000 \\
\hline BMI $\left[\mathrm{kg} / \mathrm{m}^{2}\right]$ & $29.35[25.35-34]$ & $27.1[25.1-30.7]$ & $29.8[25.6-35.1]$ & 0.279 \\
\hline \multicolumn{5}{|l|}{ Past medical history [\%] } \\
\hline Past cardiac procedure & $3[8]$ & $0[0]$ & 3 [12] & 0.540 \\
\hline Ischemic cardiomyopathy & $23[62]$ & $6[55]$ & $17[65]$ & 0.713 \\
\hline Chronic renal insufficiency & $6[16]$ & $2[18]$ & $4[15]$ & 1.000 \\
\hline Diabetes & $10[27]$ & $3[27]$ & $7[27]$ & 1.000 \\
\hline COPD & $3[8]$ & $1[9]$ & $2[8]$ & 1.000 \\
\hline Arrhythmia & $7[19]$ & $4[36]$ & $3[12]$ & 0.163 \\
\hline \multicolumn{5}{|l|}{ Preoperative support [\%] } \\
\hline Inotropes & 27 [73] & 8 [73] & 19 [73] & 1.000 \\
\hline Impella or IABP & 18 [49] & $5[45]$ & $13[50]$ & 1.000 \\
\hline ECMO & $37[100]$ & $11[100]$ & 26 [100] & 1.000 \\
\hline Ventilator & $22[59]$ & $4[36]$ & 18 [69] & 0.080 \\
\hline \multicolumn{5}{|l|}{ Preoperative hemodynamics } \\
\hline LVEF [\%] & $20.1 \pm 10.4$ & $19.7 \pm 10.4$ & $20.3 \pm 7.2$ & 0.607 \\
\hline PCWP [mm Hg] & $22.6 \pm 11.0$ & $25.0 \pm 10.7$ & $18.7 \pm 12.5$ & 0.297 \\
\hline Cardiac index [L/min/m2] & $1.8 \pm 0.5$ & $1.7 \pm 0.4$ & $1.9 \pm 0.7$ & 0.549 \\
\hline Mean PA pressure [mm Hg] & $32.6 \pm 7.0$ & $34.2 \pm 6.3$ & $30.0 \pm 8.7$ & 0.294 \\
\hline PVR [Wood units] & $3.8 \pm 2.5$ & $4.0 \pm 3.0$ & $3.5 \pm 1.9$ & 0.881 \\
\hline RA pressure $[\mathrm{mm} \mathrm{Hg}]$ & $13.8 \pm 8.8$ & $14.2 \pm 6.9$ & $13.0 \pm 13.1$ & 0.655 \\
\hline RVSWI $\left[\mathrm{mmHg} / \mathrm{mL} / \mathrm{m}^{2}\right]$ & $355.0 \pm 172.8$ & $340.2 \pm 170.7$ & $379.5 \pm 211.6$ & 0.881 \\
\hline RVSWI <400 [\%] & $6[16]$ & $4[36]$ & $2[8]$ & 0.051 \\
\hline \multicolumn{5}{|l|}{ Intended goal [\%] } \\
\hline Bridge to transplant & $26[70]$ & $7[64]$ & 19 [73] & 0.699 \\
\hline Destination therapy & 9 [24] & $2[18]$ & $7[27]$ & 0.695 \\
\hline Bridge to recovery & $2[5]$ & $2[18]$ & $0[0]$ & 0.083 \\
\hline \multicolumn{5}{|l|}{ Device Brand [\%] } \\
\hline HeartMate II & $15[41]$ & $0[0]$ & $15[58]$ & 0.001 \\
\hline HeartMate 3 & 18 [49] & 10 [91] & $8[31]$ & 0.001 \\
\hline HVAD & $4[11]$ & $1[9]$ & 3 [12] & 1.000 \\
\hline
\end{tabular}

*, values presented as no. (\%), mean $\pm \mathrm{SD}$, or median (IQR). LVAD, left ventricular assist device; ECMO, extracorporeal membrane oxygenation; CSS, complete sternal-sparing; BMI, body mass index; COPD, chronic obstructive pulmonary disease; IABP, intra-aortic balloon pump; LVEF, left ventricular ejection fraction; PCWP, pulmonary-capillary wedge pressure; PA, pulmonary artery; PVR, pulmonary vascular resistance; RA, right atrium; PAPI, pulmonary artery pulsatility index; HVAD, heart ventricular assist device. 